\title{
Preoperative assessment of lymph node metastasis in clinically node-negative rectal cancer patients based on a nomogram consisting of five clinical factors
}

\author{
Chi Zhou ${ }^{1,2,3 \#}$, Hua-Shan Liu ${ }^{1,2,3 \#}$, Xuan-Hui Liu ${ }^{1,2,3 \#}$, Xiao-Bin Zheng ${ }^{1,2,3}$, Tuo Hu ${ }^{1,2,3}$, Zhen-Xing Liang ${ }^{1,2,3}$, \\ Xiao-Wen $\mathrm{He}^{1,2}$, Xiao-Sheng $\mathrm{He}^{1,2}$, Jian-Cong $\mathrm{Hu}^{1,2}$, Xiao-Jian $\mathrm{Wu}^{1,2}$, Xian-Rui $\mathrm{Wu}^{1,2,3}$, Ping Lan ${ }^{1,2,3}$ \\ ${ }^{1}$ Department of Colorectal Surgery, ${ }^{2}$ Guangdong Provincial Key Laboratory of Colorectal and Pelvic Floor Diseases, The Sixth Affiliated Hospital \\ of Sun Yat-sen University, Guangzhou 510655, China; '3uangzhou Regenerative Medicine and Health Guangdong Laboratory, Guangzhou 510080, \\ China \\ Contributions: (I) Conception and design: C Zhou, HS Liu, XH Liu, XJ Wu, XR Wu, P Lan; (II) Administrative support: XR Wu, P Lan; (III) \\ Provision of study materials or patients: None; (IV) Collection and assembly of data: C Zhou, HS Liu, XH Liu, XB Zheng, T Hu, XW He, XS He, \\ JC Hu; (V) Data analysis and interpretation: C Zhou, HS Liu, XH Liu, XJ Wu, XR Wu, P Lan; (VI) Manuscript writing: All authors; (VII) Final \\ approval of manuscript: All authors. \\ \#These authors contributed equally to this work. \\ Correspondence to: Ping Lan, MD, PhD; Xian-Rui Wu, MD, PhD. The Sixth Affiliated Hospital, Sun Yat-sen University, 26 Yuancun Erheng Rd, \\ Guangzhou 510655, China. Email: lanping@mail.sysu.edu.cn; wuxianr5@mail.sysu.edu.cn.
}

Background: Currently, reliable approaches for accurate assessment of lymph node metastases (LNM), which is an important indication of preoperative chemoradiotherapy (CRT), are not available for clinically node-negative rectal cancer patients. This study aims to identify clinical factors associated with LNM and to establish a nomogram for LNM prediction in clinically node-negative rectal cancer patients.

Methods: The least absolute shrinkage and selection operator (LASSO) aggression and multivariate logistic regression analyses were applied to identify clinical factors associated with LNM. A nomogram was established to predict the probability of LNM in clinically node-negative rectal cancer patients based on the multivariate logistic regression model.

Results: Six potential risk factors were selected on the basis of LASSO aggression analysis, and five of them were identified as independent risk factors for LNM based on multivariate analysis, including MRI-reported tumor location, clinical T classification, MRI-reported tumor diameter, white blood cell count (WBC), and preoperative elevated tumor markers. A nomogram consisting of the five clinical factors was established and showed good discrimination. Decision curve analysis demonstrated that the established nomogram was reliable and accurate for LNM prediction in clinically node-negative rectal cancer patients.

Conclusions: A nomogram based on five clinical factors, including MRI-reported tumor location, clinical T classification, MRI-reported tumor diameter, WBC, and preoperative elevated tumor markers, are useful for assessing LNM in clinically node-negative rectal cancer patients, which is important for preoperative CRT regimens.

Keywords: Lymph node metastasis (LNM); nomogram; rectal cancer; risk factor

Submitted Jun 28, 2019. Accepted for publication Sep 10, 2019.

doi: 10.21037/atm.2019.09.127

View this article at: http://dx.doi.org/10.21037/atm.2019.09.127 


\section{Introduction}

Colorectal cancer (CRC) is the fourth leading cause of cancer-related death worldwide, and approximately $30 \%$ of CRC have been identified in the rectum (1-3). While early screenings can significantly reduce cancer morbidity (4), many cancers are diagnosed at middle or late stage, which led to a high mortality and recurrence rates. For locally advanced rectal cancer, chemoradiotherapy (CRT) prior to surgery has been established as the standard of care (5). A great amount of evidence showed that preoperative CRT significantly prevents the progress of cancers and improves overall survival of cancer patients, compared with surgery alone (6-13).

Currently, locally advanced rectal cancer is a widely accepted indication of preoperative CRT for rectal cancers $(9,14)$. Therefore, accurate preoperative staging of rectal cancer is critically important for CRC treatment. Nowadays, imaging methods including computed tomography (CT) and magnetic resonance imaging (MRI) are the main approaches to evaluate preoperative staging of CRC. MRI, due to excellent soft tissue contrast, is powerful for the identification of mesorectal node involvement as well as assessment of the circumferential resection margin (15-20). Compared to CT, which has approximately $60 \%$ of accuracy of the assessment of lymph node involvement (21), MRI showed $80 \%$ of accuracy in determining lymph node involvement in CRC patients $(15,22)$. Therefore, one fifth of RC patients with lymph node metastases (LNM) are still underdiagnosed as clinically node-negative CRC and may miss the opportunity of preoperative CRT even MRI is used for preoperative staging of CRC.

Therefore, preoperative imaging alone is insufficient for accurate evaluation of lymph node involvement in CRC patients. New tools and evaluation system are highlighted to improve the assessment of lymph node involvement in CRC patients. Numerous factors have been proposed for the assessment of LNM in previous study, such as depth of submucosal invasion, lymphovascular invasion, high-grade mucin production, signet ring features, T-stage, and poorlydifferentiated adenocarcinoma (23-29). However, most of these risk factors were based on the results from resected specimen, which were obtained postoperatively and may be inappropriate for preoperative staging of CRC.

In this study, we analyzed a number of preoperatively clinical factors, which were selected to develop a nomogram for preoperative prediction of LNM in clinically lymphnode negative rectal cancer patients. Our results are useful for physicians to screen CRC patients for preoperative CRT.

\section{Methods}

\section{Patients}

This study was approved by the Institutional Review Board (IRB) of The Sixth Affiliated Hospital of Sun Yatsen University. The training cohort of this study includes 434 clinically lymph node-negative rectal cancer patients who were hospitalized at the Sixth Affiliated Hospital of Sun Yat-sen University (Guangzhou, China) between January 2010 and December 2014. The independent validation cohort includes 165 clinically lymph nodenegative rectal cancer patients hospitalized at the same hospital between January 2015 and December 2016. Both the training and validation cohorts use the same inclusion and exclusion criteria (see below). Demographics and clinicpathological variables were prospectively maintained in the CRC Database. Both paper charts and electronic medical records were carefully reviewed.

\section{Inclusion and exclusion criteria}

The inclusion criteria were: (I) patients with rectal cancer and hospitalized at the Sixth Affiliated Hospital of Sun Yat-sen University; (II) patients with clinically nodenegative RC; (III) patients with pathological examination of a minimum of 12 lymph nodes. The exclusion criteria included: (I) patients with colon cancer; (II) patients without pre-therapy MRI images; (III) patients received preoperative CRT; (IV) patients with familiar adenomatous polyposis (FAP) or inflammatory bowel disease (IBD); (V) patients had incomplete clinical data.

\section{Variables}

Clinically node negative is defined as the short-axis diameter of lymph node $<5 \mathrm{~mm}$ based on pre-therapy MRI images. Demographic and clinic-pathological variables include general information, age at the time of surgery, race, body mass index (BMI), preoperative total protein ( $<60 v s . \geq 60 \mathrm{~g} / \mathrm{L})$, preoperative albumin $(<35 v s . \geq 35 \mathrm{~g} / \mathrm{L})$, preoperative hemoglobin $(<110 v s . \geq 110 \mathrm{~g} / \mathrm{L})$, elevated PLT $\left(>300 \times 10^{9} / \mathrm{L}\right)$, elevated WBC $\left(>10 \times 10^{9} / \mathrm{L}\right)$, elevated CEA (>5 ng/mL), elevated CA199 (>37 U/mL), MRI-based distance between tumor and anal verge, MRI-based tumor diameter, clinical $\mathrm{T}$ classification, clinical $\mathrm{N}$ classification, 
pathological $\mathrm{T}$ classification, pathological $\mathrm{N}$ classification, pathological M classification, and pathological TNM stage.

\section{Statistical analysis}

Statistical analysis was conducted using the $\mathrm{R}$ software (version 3.0.1; http://www.Rproject.org). The packages in $\mathrm{R}$ that were used in this study are reported in the data supplement. Descriptive statistics were computed for all variables. The least absolute shrinkage and selection operator (LASSO) method was used to identify predictive factors from the primary data set, weighted by their respective coefficients. Multivariate analyses of selected suboptimal risk factors associated with LNM were conducted using the logistic regression analysis. Bidirectional elimination was done to fit regression models based on the lowest Akaike information criterion. A prediction nomogram was established based on multivariate logistic regression analysis. Decision curve analysis was conducted to determine the clinical usefulness of the nomogram by quantifying the net benefits at different threshold probabilities. $\mathrm{P}$ values less than 0.05 were considered statistically significant.

\section{Results}

\section{Patient characteristics}

A total of 599 clinically lymph node-negative rectal cancer patients were enrolled in this study, including 511 patients (85.3\%) underwent laparoscopic surgery and 88 (14.7\%) underwent open surgery. The training cohort included 434 patients while the independent validation cohort included 165 patients. LNM was found in $24.2 \%$ and $30.9 \%$ of the training and validation cohorts, respectively $(\mathrm{P}=0.094)$. Preoperative clinical factors including age, gender, BMI, CEA, CA199, total protein, albumin, hemoglobin, PLT, WBC, MRI-based tumor location, MRIbased tumor diameter and clinical $\mathrm{T}$ classification were shown in Table 1.

\section{Risk factors for LNM}

Risk factors for LNM in clinically node-negative rectal cancer patients were identified using multivariate logistic regression models of LASSO method. Of 13 clinical factors, six potential predictors were selected from 434 patients in the training cohort, based on nonzero coefficients in the
LASSO logistic regression model (Figure 1). In order to optimize the predictive model, the six potential predictors were further evaluated using multivariate logistic regression model. The multivariate logistic regression analysis revealed that MRI-based tumor location $(\mathrm{P}<0.001)$, clinical $\mathrm{T}$ classification $(\mathrm{P}<0.001)$, MRI-based tumor diameter $(\mathrm{P}<0.001)$, preoperative WBC $(\mathrm{P}=0.048)$ and preoperative elevated tumor markers $(\mathrm{P}=0.005)$ were independent LNM risk factors for clinically node-negative rectal patients (Figure 2).

\section{Development and validation of an individualized prediction model}

A nomogram based on the five independent risk factors was established (Figure 3). When using, a vertical line is drawn for each variable to see their respective score. Each score was added together to get the total score, which determines the probability of LNM. For example, a patient with T3/T4 classification (points=32), with tumor located at $6 \mathrm{~cm}$ above from anal verge (points=39), with a tumor diameter of $10 \mathrm{~mm}$ (points $=50$ ), without elevated tumor marker (points=0), with elevated preoperative WBC (points=35) would have a total score of 156 , and a predicted LNM risk of $75 \%$.

The area under ROC curve (AUC) of the model was 0.743 (95\% CI: 0.691-0.795), which was greater than MRIbased tumor location (0.673, 95\% CI: $0.620-0.727)$, clinical T classification (0.626, 95\% CI: 0.567-0.685), MRI-based tumor diameter $(0.630,95 \%$ CI: $0.568-0.692)$, preoperative WBC $(0.524,95 \%$ CI: $0.460-0.589)$ and preoperative elevated tumor markers $(0.588,95 \%$ CI: $0.522-0.654)$ in the training cohorts, suggesting a better predictive value (Figure 4). The AUC of the model was shown to be 0.777 (95\% CI: $0.705-0.848$ ) in the validation cohort, which further confirmed the predictive value (Figure 4) The calibration graph showed favorable agreement between prediction and observation for both the training and validation cohorts because the calibration curve was close to the 45-degree line, which suggests that the model can perfectly predict the real event as shown in Figure 4.

\section{Clinical applications}

The decision curve analysis of the nomogram showed that the nomogram adds more benefit to LNM prediction in clinically node-negative rectal cancer patients than either the treat-all-patients scheme or the treat-none scheme when 
Table 1 Patient characteristics

\begin{tabular}{|c|c|c|}
\hline Characteristics & $\begin{array}{c}\text { The training } \\
\text { cohort }\end{array}$ & $\begin{array}{c}\text { The validation } \\
\text { cohort }\end{array}$ \\
\hline Number of patients & 434 & 165 \\
\hline LNM & $105(24.2)$ & $51(30.9)$ \\
\hline Age (years) & $59.1 \pm 12.7$ & $59.8 \pm 11.7$ \\
\hline \multicolumn{3}{|l|}{ Gender } \\
\hline Female & $183(42.2)$ & $65(39.4)$ \\
\hline Male & $251(57.8)$ & $100(60.6)$ \\
\hline Body mass index $\left(\mathrm{kg} / \mathrm{m}^{2}\right)$ & $22.7 \pm 3.4$ & $22.7 \pm 3.2$ \\
\hline \multicolumn{3}{|l|}{ CEA level (ng/mL) } \\
\hline$\leq 5$ & 318 (73.3) & $119(72.1)$ \\
\hline$>5$ & $116(26.7)$ & $46(27.9)$ \\
\hline \multicolumn{3}{|l|}{ CA199 level (U/mL) } \\
\hline$\leq 37$ & $395(91.0)$ & $153(92.7)$ \\
\hline$>37$ & $39(9.0)$ & $12(7.3)$ \\
\hline \multicolumn{3}{|c|}{ Preoperative total protein (g/L) } \\
\hline$<60$ & $26(6.0)$ & $10(6.1)$ \\
\hline$\geq 60$ & $408(94)$ & $155(93.9)$ \\
\hline \multicolumn{3}{|l|}{ Preoperative albumin (g/L) } \\
\hline$<35$ & $13(3.0)$ & $7(4.2)$ \\
\hline$\geq 35$ & $421(97.0)$ & $158(95.8)$ \\
\hline
\end{tabular}

Table 1 (continued)

the threshold probability of a patient or doctor is between $5 \%$ and $70 \%$ (Figure 5 ). The true and false positive rates in each risk threshold were also shown in Figure 5.

\section{Discussion}

Preoperative CRT is a standard regimen for advanced rectal cancer patients due to its prominent advantages on local control as well as overall survival $(6,7,13)$. The widely accepted clinical indications for preoperative CRT are rectal cancer patients with lymph node involvement $(9,14)$. Therefore, assessment of accurate preoperative staging of rectal cancer patients is crucial for clinical decision-making.

MRI is recommended as the best imaging approach for clinical staging of rectal cancer (15). However, MRI, which is not capable of detecting metastatic lymph nodes $<3 \mathrm{~mm}$, exhibits an accuracy rate of $80 \%$ for predicting lymph node
Table 1 (continued)

\begin{tabular}{lcc}
\hline Characteristics & $\begin{array}{c}\text { The training } \\
\text { cohort }\end{array}$ & $\begin{array}{c}\text { The validation } \\
\text { cohort }\end{array}$ \\
\hline Preoperative hemoglobin $(\mathrm{g} / \mathrm{L})$ & $53(12.2)$ & $26(15.8)$ \\
$<110$ & $381(87.8)$ & $139(84.2)$ \\
$\geq 110$ & & \\
Preoperative PLT (10\%) & & \\
$\leq 300$ & $386(88.9)$ & $140(84.8)$ \\
$>300$ & $48(11.1)$ & $25(15.2)$ \\
Preoperative WBC (10\%) & & $155(93.9)$ \\
$\leq 10$ & $417(96.1)$ & $10(6.1)$ \\
$>10$ & $17(3.9)$ & $5.8 \pm 2.8$ \\
Location (cm) & $5.0 \pm 2.8$ & $3.2 \pm 1.5$ \\
Tumor diameter (cm) & $5.4 \pm 3.5$ & \\
Clinical T classification & & $21(12.7)$ \\
T1 & $37(8.5)$ & $41(24.8)$ \\
T2 & $145(33.4)$ & $100(60.6)$ \\
T3 & $230(53.0)$ & $3(1.8)$ \\
T4 & $22(5.1)$ & \\
\hline
\end{tabular}

CA199, carbohydrate antigen 19-9; CEA, carcinoembryonic antigen; LNM, lymph node metastasis; location, MRI-based distance between tumor and anal verge; PLT, platelet; tumor diameter, MRI-based diameter of tumor; WBC, white blood cell.

involvement $(15,22)$. In this study, the false negative rate of MRI for predicting lymph node involvement ranged from $20 \%$ to $30 \%$, which is consistent with previous reports. The limitations of existing imaging tools may exclude prospective patients from CRT because of missing diagnoses of LNM. Therefore, finding other methods to assess accurate status of regional lymph node involvement in rectal cancer patients is of great importance. In the present study, we aimed to establish a clinical scoring system based on preoperative parameters to accurately assess the status of lymph nodes in clinically node-negative rectal cancer patients.

Nomograms have been widely used to visualize risk factors and prognosis in CRC patients $(30,31)$. In our study, six potential predictors from 13 risk factor candidates were used to establish a nomogram by shrinking the regression coefficients using the LASSO method, which has been 

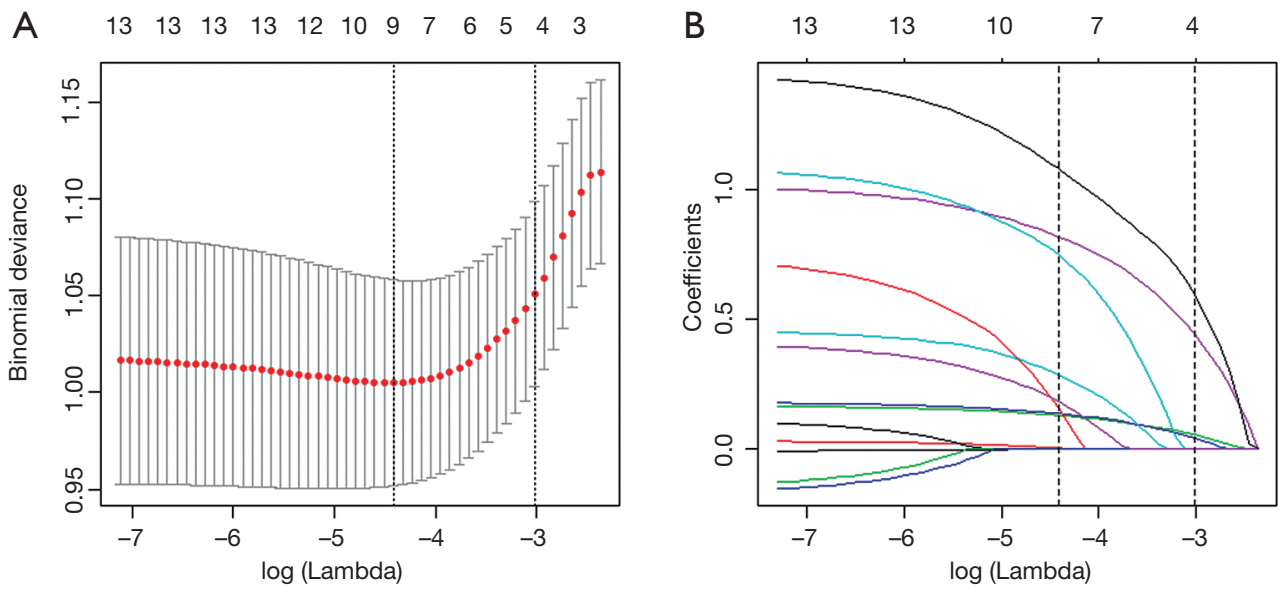

Figure 1 Suboptimal factor selection using the least absolute shrinkage and selection operator (LASSO) binary logistic regression model. (A) Tuning parameter (Lambda) selection in the LASSO model used 10-fold cross-validation via minimum criteria. The Binomial Deviance was plotted versus $\log$ (Lambda). Dotted vertical lines were drawn at the optimal values using the minimum criteria and the 1 standard error of the minimum criteria (the 1-SE criteria). (B) LASSO coefficient profiles of the 13 texture features. A coefficient profile plot was generated against the $\log$ (Lambda) sequence. Vertical line represents the values selected using 10 -fold cross-validation, where optimal lambda resulted in 6 nonzero coefficients.

Clinical T stage

$\mathrm{T} 1 / \mathrm{T} 2$

$\mathrm{T} 3 / \mathrm{T} 4$

Location, cm

Diameter, cm

TumorMarker

0

1

2

WBC, $10 \%$

$\leq 10$

$>10$

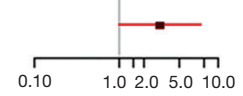

Reference

(3.02, 0.98 to 9.02$)$

0.048

Figure 2 Forest plot of multivariable logistic model associations with LNM in clinically node-negative rectal cancer patients. Location: MRI-based distance between tumor and anal verge; tumor diameter: MRI-based diameter of tumor; tumor marker: 0= normal CEA and CA199, 1= elevated CEA or CA199, 2= elevated CEA and CA199; LNM, lymph node metastases; MRI, magnetic resonance imaging; WBC, white blood cell.

Reference

$\begin{array}{ll}(2.65,1.57,4.60) & <0.001 \\ (1.18,1.08 \text { to } 1.29) & <0.001 \\ (1.17,1.10 \text { to } 1.26) & <0.001\end{array}$

Points

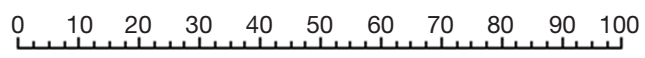

Location

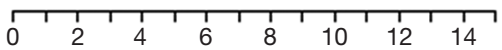

cT

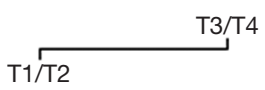

Diameter

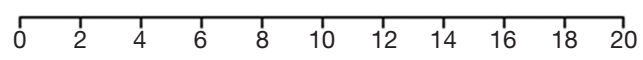

TumorMarker

PreopWBC
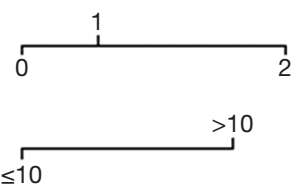

Total points

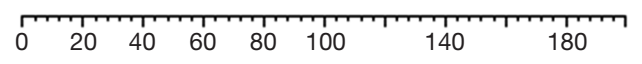

Risk of positive $\mathrm{N}$

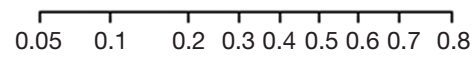

Figure 3 The nomogram for predicting LNM in clinically nodenegative rectal cancer patients. The nomogram was established in the training cohort using multivariable regression, consisting of the location, clinical T classification, tumor diameter, WBC and tumor marker. LNM, lymph node metastases; WBC, white blood cell. 

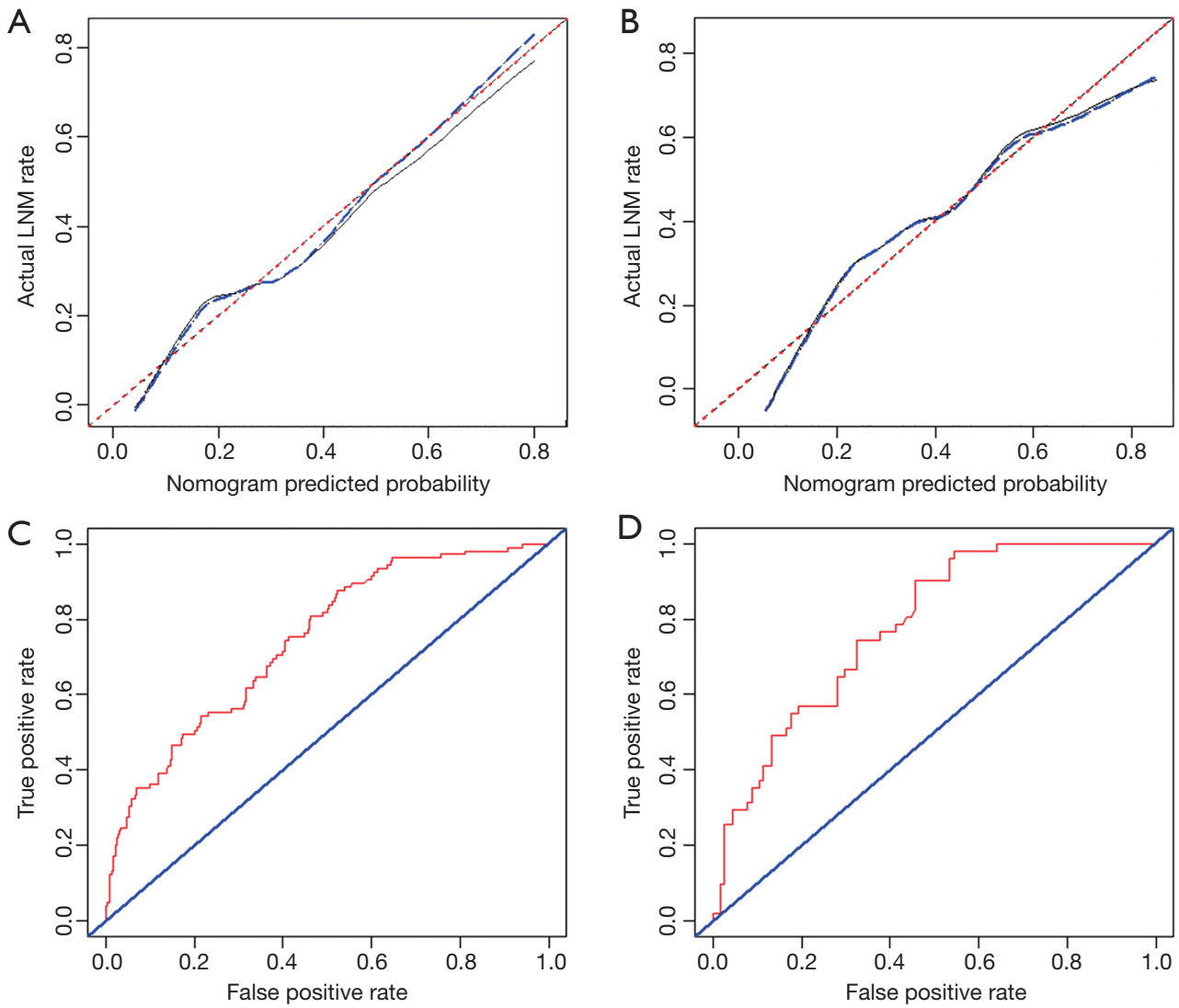

Figure 4 (A,B) Calibration curves of the developed nomogram in each cohort. (A) Calibration curve of the nomogram in the training cohort. (B) Calibration curve of the nomogram in the validation cohort. The x-axis represents the predicted probability from the nomogram, and the $y$-axis is the actual probability of LNM in clinically node-negative rectal cancer patients. The dashed line indicates that predicted outcome perfectly corresponds with actual outcome. The blue line indicates the bias-corrected accuracy of the nomogram while a closer fit to the diagonal dotted line represents a better prediction. (C,D) Receiver operating characteristic (ROC) curve. (C) ROC curve in the training cohort [area under the curve (AUC): 0.743]. (D) ROC curve in the validation curve (AUC: 0.777). Diagonal segments are produced by ties. LNM, lymph node metastases.

recommended for variable selection $(21,32)$. Then, the multivariate logistic regression model was used for the determination of the optimal predictors $(33,34)$.

T classification, which is an index of the depth of tumor invasion, has been well recognized as one of the most important predictors of LNM for CRC (24,35-38). A study based on 804 cases reported that the percentage of lymph node involvement arises from $5.7 \%$ to $78.8 \%$ accordingly when the $T$ classification increases from 1 to 4 (36). In our study, we found that the clinical $\mathrm{T}$ classification is a significant independent predictor of LNM in clinically node-negative rectal cancer patients, which is consistent with previous studies.

The diameter of tumor or the tumor size was also a
LNM predictor in our nomogram, which was in accordance with the study conducted by Zhang et al. (39). While several studies failed to identify a direct association between tumor size and LNM, they demonstrated an association between tumor size and tumor progression. For example, Cai et al. (40) reported that the tumor size was significantly related to local advancement of tumors. Chok and colleagues (41) also reported that the tumor size was associated with pT classification. Furthermore, a study conducted by Kikuchi et al. (42) found a direct correlation between tumor diameter and the depth of tumor invasion in 182 CRC cases. Therefore, increased diameter of tumor, which is an independent prognostic factor of tumor progression, is also relatively associated with LNM. 

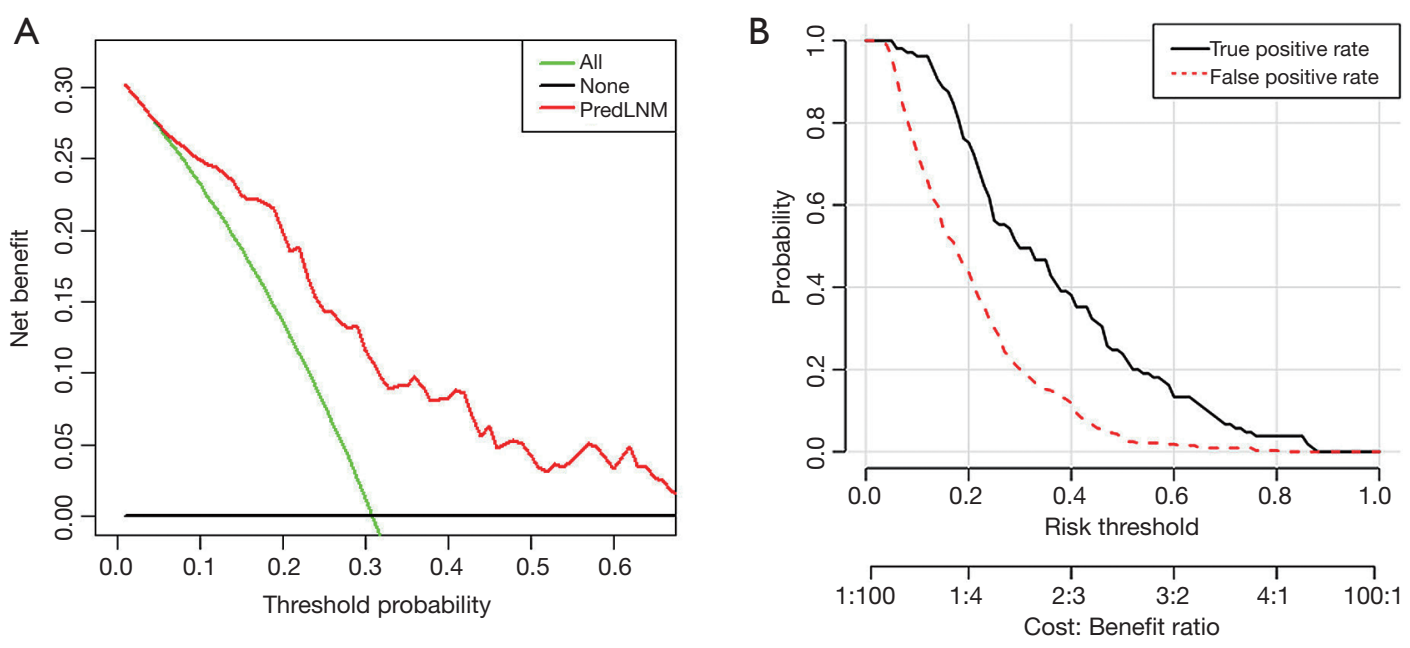

Figure 5 (A) Decision curve analysis of the predicted nomogram model. The Y-axis measures the net benefit. The red line represents the predicted nomogram model. The green line represents the assumption that all clinically node-negative RC patients had LMN. Thin black line represents the assumption that no patients had LMN. The net benefit was calculated by subtracting the proportion of all patients who are false positive from the proportion who are true positive, weighting by the relative harm of forgoing treatment compared with the negative consequences of an unnecessary treatment. The decision curve showed that the nomogram adds more benefit than either the treatall-patients scheme or the treat-none scheme to predicting postoperative AL when the threshold probability of a patient or doctor ranges between $10 \%$ and $67 \%$. (B) The true positive rates and false positive rates in each risk thresholds. The black light represents the true positive rates of the model, while the red light represents the false one. LNM, lymph node metastases.

CEA and CA19-9, which are the most common tumor markers of CRC, have been currently utilized in clinical practice for cancer screening and follow-up $(43,44)$. Previous studies have fully demonstrated that increased levels of CEA and CA19-9 were risk factors of tumor recurrence and anti-tumor agent resistance (45). A study based on 130 CRC patients revealed that elevated CEA and CA19-9 were significantly correlated with lymph nodes involvement (45). In the present study, our results also suggest that the elevation of these two preoperative tumor markers were predictors for LNM.

The location of tumor also served as a prognostic factor of LNM in our study. Longer distance between tumor and the anal verge is a risk factor of LNM. To the best of our knowledge, only a few studies focus on the association between tumor location and LNM. Two studies $(46,47)$ even demonstrated the opposite results. Therefore, it is difficult to draw a conclusion on the relationship between tumor location and LNM risk based on currently available studies. Further studies are highlighted to carefully assess the exact relationship between the location of tumor and LNM.

The white blood cell count (WBC) was a widely used marker of infection and inflammation (48). Numerous studies have demonstrated that inflammation is involved in tumorigenesis and metastasis (49), mainly through changing the systemic immune status and local microenvironment (50), which is partially reflected by the abnormalities of WBC count (51). Several studied have found that more WBCs were associated with carcinogenesis, tumor progression and cancer mortality (48,51-53). Our study suggests that elevated WBC level was an ominous prognostic sign of LNM.

While several studies evaluated predictors for LNM, no study focused on the prediction of LNM in clinically nodenegative rectal cancer patients. In this study, we established a nomogram consisting of 5 clinical factors. The decision curve showed that our nomogram is better than either the treat-all-patients scheme or the treat-none scheme in LNM prediction when the threshold probability of a patient or doctor ranges between $5 \%$ and $70 \%$. The nomogramderived probability was also calculated for better usage. For example, the sensitivity and specificity were $75 \%$ and $56 \%$, respectively, in the training cohort, and $90 \%$ and $45 \%$, respectively in the validation cohort, when a $20 \%$ risk of LMN was set as the nomogram-derived probability cut-offs. These results suggest the high sensitivity and specificity of our nomogram in identifying CRC patients who need CRT. 
Thus, our nomogram, which has up to $75 \%$ of accuracy of detecting LNM, provides supplemental information for individual clinical decision, which can be used to properly enroll CRC patients for CRT.

There are several limitations in our study. For instance, our study did not fully take advantages of the MRI examination. Recently, radiomics, which incorporate the features of imaging examination, has emerged in the field of cancer research (21). The nomogram including imaging features may be more convincing than using the result of MRI examination alone. Besides, $30 \%$ of rectal cancer patients were initially considered to be nodepositive but were negative in the pathology report, and these patients might be overtreated (21). Therefore, it is of great importance to develop models for preoperative individualized assessment of LN metastasis in clinically node-positive patients, which requires our further investigation.

In conclusion, we established a nomogram consisting of five preoperative clinical factors for individualized prediction of LNM in clinically node-negative rectal cancer patients. Our nomogram is useful for enrolling CRC patients for CRT.

\section{Acknowledgments}

Funding: This work was supported by National Key R\&D Program of China (No. 2017YFC1308800), National Natural Science Foundation of China (No. 81870383), Clinical Innovation Research Program of Guangzhou Regenerative Medicine and Health Guangdong Laboratory (No. 2018GZR0201005), Guangdong Natural Science Foundation (No. 2017A030313785), Science and Technology Planning Project of Guangzhou City (No. 201804010014), Science and Technology Planning Project of Guangdong Province (No. 2015B020229001 and 20160916), and National Key Clinical Discipline.

\section{Footnote}

Conflicts of Interest: The authors have no conflicts of interest to declare.

Ethical Statement: The authors are accountable for all aspects of the work in ensuring that questions related to the accuracy or integrity of any part of the work are appropriately investigated and resolved. This study was approved by the Institutional Review Board (IRB) of The
Sixth Affiliated Hospital of Sun Yat-sen University. All patients provided written informed consent to participate in this study.

\section{References}

1. Siegel RL, Miller KD, Jemal A. Cancer statistics, 2016. CA Cancer J Clin 2016;66:7-30.

2. Global Burden of Disease Cancer Collaboration, Fitzmaurice C, Dicker D, et al. The Global Burden of Cancer 2013. JAMA Oncol 2015;1:505-27.

3. Chen $W$, Zheng R, Baade PD, et al. Cancer statistics in China, 2015. CA Cancer J Clin 2016;66:115-32.

4. Cancer Prevention Overview (PDQ(R)): Patient Version. PDQ Cancer Information Summaries. Bethesda (MD), 2002.

5. Sineshaw HM, Jemal A, Thomas CR Jr, et al. Changes in treatment patterns for patients with locally advanced rectal cancer in the United States over the past decade: An analysis from the National Cancer Data Base. Cancer 2016;122:1996-2003.

6. Swedish Rectal Cancer Trial, Cedermark B, Dahlberg M, et al. Improved survival with preoperative radiotherapy in resectable rectal cancer. N Engl J Med 1997;336:980-7.

7. Sauer R, Becker H, Hohenberger W, et al. Preoperative versus postoperative chemoradiotherapy for rectal cancer. N Engl J Med 2004;351:1731-40.

8. Minsky BD. Is preoperative chemoradiotherapy still the treatment of choice for rectal cancer? J Clin Oncol 2009;27:5115-6.

9. Tural D, Ozturk M, Selcukbiricik F, et al. Preoperative chemoradiotherapy improves local recurrence free survival in locally advanced rectal cancer. J BUON 2013;18:385-90.

10. Kalyan A, Rozelle S, Benson A 3rd. Neoadjuvant treatment of rectal cancer: where are we now? Gastroenterol Rep (Oxf) 2016;4:206-9.

11. Li Y, Wang J, Ma X, et al. A Review of Neoadjuvant Chemoradiotherapy for Locally Advanced Rectal Cancer. Int J Biol Sci 2016;12:1022-31.

12. Rödel C, Martus P, Papadoupolos T, et al. Prognostic significance of tumor regression after preoperative chemoradiotherapy for rectal cancer. J Clin Oncol 2005;23:8688-96.

13. Delaney CP, Lavery IC, Brenner A, et al. Preoperative radiotherapy improves survival for patients undergoing total mesorectal excision for stage T3 low rectal cancers. Ann Surg 2002;236:203-7. 
14. Weiser MR, Zhang Z, Schrag D. Locally advanced rectal cancer: time for precision therapeutics. Am Soc Clin Oncol Educ Book 2015:e192-6.

15. Group MS. Extramural depth of tumor invasion at thinsection MR in patients with rectal cancer: results of the MERCURY study. Radiology 2007;243:132-9.

16. Iafrate F, Laghi A, Paolantonio P, et al. Preoperative staging of rectal cancer with MR Imaging: correlation with surgical and histopathologic findings. Radiographics 2006;26:701-14.

17. Kaur H, Choi H, You YN, et al. MR imaging for preoperative evaluation of primary rectal cancer: practical considerations. Radiographics 2012;32:389-409.

18. Smith N, Brown G. Preoperative staging of rectal cancer. Acta Oncol 2008;47:20-31.

19. Guillem JG, Diaz-Gonzalez JA, Minsky BD, et al. c'T3N0 rectal cancer: potential overtreatment with preoperative chemoradiotherapy is warranted. J Clin Oncol 2008;26:368-73.

20. Torkzad MR, Pahlman L, Glimelius B. Magnetic resonance imaging (MRI) in rectal cancer: a comprehensive review. Insights Imaging 2010;1:245-67.

21. Huang YQ, Liang CH, He L, et al. Development and Validation of a Radiomics Nomogram for Preoperative Prediction of Lymph Node Metastasis in Colorectal Cancer. J Clin Oncol 2016;34:2157-64.

22. Park JS, Jang YJ, Choi GS, et al. Accuracy of preoperative MRI in predicting pathology stage in rectal cancers: nodefor-node matched histopathology validation of MRI features. Dis Colon Rectum 2014;57:32-8.

23. Mou S, Soetikno R, Shimoda T, et al. Pathologic predictive factors for lymph node metastasis in submucosal invasive (T1) colorectal cancer: a systematic review and meta-analysis. Surg Endosc 2013;27:2692-703.

24. Kitajima K, Fujimori T, Fujii S, et al. Correlations between lymph node metastasis and depth of submucosal invasion in submucosal invasive colorectal carcinoma: a Japanese collaborative study. J Gastroenterol 2004;39:534-43.

25. The Paris endoscopic classification of superficial neoplastic lesions: esophagus, stomach, and colon: November 30 to December 1, 2002. Gastrointest Endosc 2003;58:S3-43.

26. Glasgow SC, Bleier JI, Burgart LJ, et al. Meta-analysis of histopathological features of primary colorectal cancers that predict lymph node metastases. J Gastrointest Surg 2012;16:1019-28.

27. Nissan A, Stojadinovic A, Shia J, et al. Predictors of recurrence in patients with T2 and early T3, N0 adenocarcinoma of the rectum treated by surgery alone. $\mathrm{J}$
Clin Oncol 2006;24:4078-84.

28. Blumberg D, Paty PB, Picon AI, et al. Stage I rectal cancer: identification of high-risk patients. J Am Coll Surg 1998;186:574-9; discussion 579-80.

29. Talbot IC, Ritchie S, Leighton M, et al. Invasion of veins by carcinoma of rectum: method of detection, histological features and significance. Histopathology 1981;5:141-63.

30. Li Q, Dai W, Li Y, et al. Nomograms for predicting the prognostic value of serological tumor biomarkers in colorectal cancer patients after radical resection. Sci Rep 2017;7:46345.

31. Sun Y, Lin H, Lu X, et al. A nomogram to predict distant metastasis after neoadjuvant chemoradiotherapy and radical surgery in patients with locally advanced rectal cancer. J Surg Oncol 2017;115:462-9.

32. Liang C, Huang Y, He L, et al. The development and validation of a CT-based radiomics signature for the preoperative discrimination of stage I-II and stage III-IV colorectal cancer. Oncotarget 2016;7:31401-12.

33. Lee TF, Chao PJ, Chang L, et al. Developing Multivariable Normal Tissue Complication Probability Model to Predict the Incidence of Symptomatic Radiation Pneumonitis among Breast Cancer Patients. PLoS One 2015;10:e0131736.

34. Lee TF, Chao PJ, Ting HM, et al. Using multivariate regression model with least absolute shrinkage and selection operator (LASSO) to predict the incidence of Xerostomia after intensity-modulated radiotherapy for head and neck cancer. PLoS One 2014;9:e89700.

35. Resch A, Langner C. Risk assessment in early colorectal cancer: histological and molecular markers. Dig Dis 2015;33:77-85.

36. Sitzler PJ, Seow-Choen F, Ho YH, et al. Lymph node involvement and tumor depth in rectal cancers: an analysis of 805 patients. Dis Colon Rectum 1997;40:1472-6.

37. Son HJ, Song SY, Lee WY, et al. Characteristics of early colorectal carcinoma with lymph node metastatic disease. Hepatogastroenterology 2008;55:1293-7.

38. Ding PR, An X, Cao Y, et al. Depth of tumor invasion independently predicts lymph node metastasis in T2 rectal cancer. J Gastrointest Surg 2011;15:130-6.

39. Zhang H, Chen CS, Cong JC, et al. Clinicopathological characteristics of advanced colorectal cancer $30 \mathrm{~mm}$ or smaller in diameter. Chin Med Sci J 2007;22:98-103.

40. Cai G, Xu Y, Zhu X, et al. A simple scoring system based on clinical features to predict locally advanced rectal cancers. J Gastrointest Surg 2009;13:1299-305.

41. Chok KS, Law WL. Prognostic factors affecting survival 
and recurrence of patients with p T1 and p'T2 colorectal cancer. World J Surg 2007;31:1485-90.

42. Kikuchi R, Takano M, Takagi K, et al. Management of early invasive colorectal cancer. Risk of recurrence and clinical guidelines. Dis Colon Rectum 1995;38:1286-95.

43. Yamamoto H, Miyake Y, Noura S, et al. Tumor markers for colorectal cancer. Gan To Kagaku Ryoho 2001;28:1299-305.

44. Fang WL, Chang SC, Lin JK, et al. Metastatic potential in T1 and T2 colorectal cancer. Hepatogastroenterology 2005;52:1688-91.

45. Nozoe T, Rikimaru T, Mori E, et al. Increase in both CEA and CA19-9 in sera is an independent prognostic indicator in colorectal carcinoma. J Surg Oncol 2006;94:132-7.

46. Nascimbeni R, Burgart LJ, Nivatvongs S, et al. Risk of lymph node metastasis in T1 carcinoma of the colon and rectum. Dis Colon Rectum 2002;45:200-6.

47. Steup WH, Moriya Y, van de Velde CJ. Patterns of lymphatic spread in rectal cancer. A topographical analysis on lymph node metastases. Eur J Cancer 2002;38:911-8.

48. Margolis KL, Rodabough RJ, Thomson CA, et al.

Cite this article as: Zhou C, Liu HS, Liu XH, Zheng XB, Hu T, Liang ZX, He XW, He XS, Hu JC, Wu XJ, Wu XR, Lan P. Preoperative assessment of lymph node metastasis in clinically node-negative rectal cancer patients based on a nomogram consisting of five clinical factors. Ann Transl Med 2019;7(20):543. doi: 10.21037/atm.2019.09.127
Prospective study of leukocyte count as a predictor of incident breast, colorectal, endometrial, and lung cancer and mortality in postmenopausal women. Arch Intern Med 2007;167:1837-44.

49. Kapp DS, Fischer D, Gutierrez E, et al. Pretreatment prognostic factors in carcinoma of the uterine cervix: a multivariable analysis of the effect of age, stage, histology and blood counts on survival. Int J Radiat Oncol Biol Phys 1983;9:445-55.

50. Mantovani A, Allavena P, Sica A, et al. Cancer-related inflammation. Nature 2008;454:436-44.

51. Lee YJ, Lee HR, Nam CM, et al. White blood cell count and the risk of colon cancer. Yonsei Med J 2006;47:646-56.

52. Cho Y, Kim KH, Yoon HI, et al. Tumor-related leukocytosis is associated with poor radiation response and clinical outcome in uterine cervical cancer patients. Ann Oncol 2016;27:2067-74.

53. Kasuga I, Makino S, Kiyokawa H, et al. Tumor-related leukocytosis is linked with poor prognosis in patients with lung carcinoma. Cancer 2001;92:2399-405. 


\section{Supplementary}

\#Related Computerized Programs for the Nomogram Using R in Current Study

\#For the least absolute shrinkage and selection operator (LASSO) binary logistic regression mode

library(glmnet)

fit<-glmnet(model.matrix ( Age+Gender......,data=training_data), as.matrix(PositiveN,data=training_data),family="binomial",alpha=1) cv.fit<-cv.glmnet(model.matrix ( Age+Gender......,data=training_data), as.matrix(PositiveN,data=training_data),family="binomial",alp ha=l)

predict(cv.fit,type='coefficients',s=cv.fit\$lambda.min)

plot(cv.fit)

plot(cv.fit\$glmnet.fit,xvar ="lambda")

\#For Nomogram

library(rms)

$\mathrm{f}<-\operatorname{lrm}$ (PositiveLNM $\sim$ Location+ClinicalTstage......, $\mathrm{x}=\mathrm{T}, \mathrm{y}=\mathrm{T}$ )

nom <- nomogram(f, fun=plogis, fun.at=c(.05, seq(.1,.9, by=.1), .95, l),lp=F,maxscale=100, funlabel="Risk of PositiveLNM")

plot(nom)

\#For Resampling Validation of Nomogram

validate(f,method="boot",B=1000,dxy=T)

\#For Computing the AUC(C-Index) and 95\% CI

library(ROCR)

pred<-prediction(pre,PositiveLNM)

performance(pred,'auc')

$\mathrm{SE}<-\mathrm{SD} / 2 ; 95 \% \mathrm{CI}=\mathrm{AUC}(\mathrm{C}-$ Index $) \pm 1.96 \times \mathrm{SE}$

\#For Calibration Curve

plot(calibrate(f,method="boot",B=1000),scatld.opts=list(nhistSpike=240, ,ide $=1$, frac $=0.08$,lwd=1,nint=50))

lines(calibrate (f,method="boot",B=1000), lwd=2,lty=5, $\mathrm{col}=\mathrm{c}(\mathrm{rgb}(0,0,255$, maxColorValue $=255))$ )

abline $(0,1$, lty $=3, \mathrm{lwd}=2, \mathrm{col}=\mathrm{c}(\mathrm{rgb}(255,0,0, \max$ ColorValue $=255)))$

\#For External Validation of Nomogram

$\mathrm{fl}<-\operatorname{lrm}($ PositiveLNM $\sim$ predict(f, data=validation_data), $\mathrm{x}=\mathrm{T}, \mathrm{y}=\mathrm{T}$ )

validate $(\mathrm{fl}$, method="boot",B=1000, $\mathrm{dxy}=\mathrm{T}$ )

\#For the Calibration Curve of the Validation Cohort

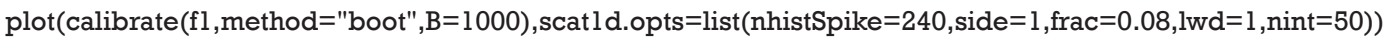

lines(calibrate $(\mathrm{fl}$, method="boot", $\mathrm{B}=1000), \mathrm{lwd}=2, \mathrm{lty}=5, \mathrm{col}=\mathrm{c}(\mathrm{rgb}(0,0,255$, maxColorValue $=255))$

abline $(0,1$, lty $=3, \operatorname{lwd}=2, \mathrm{col}=\mathrm{c}(\mathrm{rgb}(255,0,0, \max$ ColorValue $=255)))$

\#For Computing the AUC(C-Index) and 95\% CI of the Validation Cohort

fl <- glm(PositiveLNM predict(f, data=validation_data),family=binomial(link='logit'), data=validation_data)

pre<-predict(fl,type='response')

pred<-prediction(pre,PositiveLNM)

performance(pred,'auc')

$\mathrm{SE}<-\mathrm{SD} / 2 ; 95 \% \mathrm{CI}=\mathrm{AUC}(\mathrm{C}-$ Index $) \pm 1.96 \times \mathrm{SE}$

\#For the Decision Curve of the Validation Cohort

library(MASS)

validation_data\$PositiveLNM = predict(fl, type="response")

plot(dca(data=validation_data, outcome="PositiveN", predictors="PositiveLNM", smooth="TRUE",xstop=1)\$net.benefit.threshold, km\$net. benefit.none, type $=$ "l", lwd=2, xlim $=c(0,1)$, ylim $=c(0,0.32), x l a b=$ "Threshold Probability",ylab = "Net Benefit") 